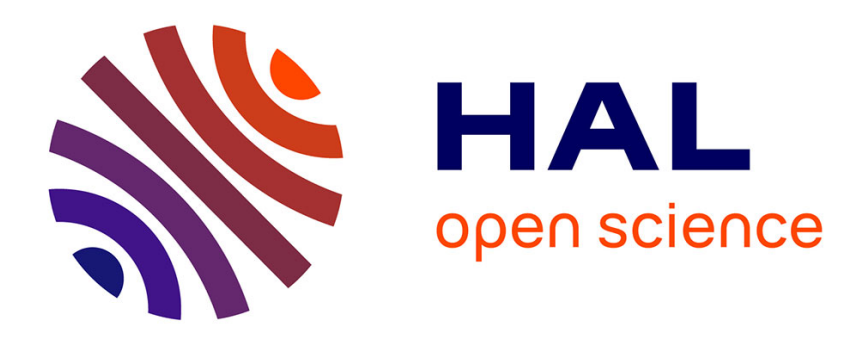

\title{
A Proof of Stability of Model-Free Control Emmanuel Delaleau
}

\section{To cite this version:}

Emmanuel Delaleau. A Proof of Stability of Model-Free Control. 2014 IEEE Conference on Norbert Wiener in the 21st Century (21CW), Jun 2014, Boston, MA, United States. 10.1109/NORBERT.2014.6893908 . hal-02318645

\section{HAL Id: hal-02318645 \\ https://hal.science/hal-02318645}

Submitted on 17 Oct 2019

HAL is a multi-disciplinary open access archive for the deposit and dissemination of scientific research documents, whether they are published or not. The documents may come from teaching and research institutions in France or abroad, or from public or private research centers.
L'archive ouverte pluridisciplinaire HAL, est destinée au dépôt et à la diffusion de documents scientifiques de niveau recherche, publiés ou non, émanant des établissements d'enseignement et de recherche français ou étrangers, des laboratoires publics ou privés. 


\title{
A Proof of Stability of Model-Free Control
}

\author{
Emmanuel Delaleau \\ Institut supérieur de l'électronique et du numérique \\ 20 rue Cuirassé Bretagne, F-29 228 Brest (cedex), France \\ Email: emmanuel.delaleaudisen.fr
}

\begin{abstract}
Cybernetics involves Control Theory and Control Practice. From its roots, Cybernetics has always been intimately to Control. The paper is devoted to the proof of an important theorem for the development of control: the closed loop stability of control laws that are calculated in the framework of modelfree control. Everyone knows the importance of control in the field of Cybernetics [1].
\end{abstract}

\section{INTRODUCTION}

This new framework has been recently introduced in France [2], [3] and has led to several convincing applications. The idea underlying this methodology is the estimation [4] of some derivatives of measured variables in order to use them in the estimation of the not-modeled effects that influence the to-be-controlled system and to use this information in the feedback loop in order to cancel undesired effects. The control is based on a tracking of the response of an auxiliary model fed by algebraic estimations relying on measurements to which a given behavior is impressed.

Most nonlinear control laws are implemented using asymptotic observers. Contrarily to the linear case, the separation principle is not valid in the case of nonlinear control systems. However, most nonlinear control law methodologies have stability proves, most of them rely on standard tools coming from the pioneer work of Lyapunov. All these proofs need a model and that is a reason why there is no general proof of stability of model-free control.

The present paper aims at filling this gap and using advanced tools for stability analysis [5]-[8] will present a general proof of stability of model-free Control. These tools have already been used for the proof of stability and robustness of exact feed-forward linearization [9]-[12]. We think that this result is important for the development of Cybernetics and the teaching of control.

The paper is organized as follow:

- Section II recalls the main aspects and ingredients of model-free control: the development of algebraic estimator (Sec. II-B) and the original development of model-free control (Sec. II-C).

- Section III exposes in details a continuous-time reformulation of model-free control which is the first contribution of this work. MFC is by essence a discrete-time control law although it is applied to continuous-time system. The continuous-time version of the model-free control law allow to draw a stability analysis of the tracking error.

- $\quad$ Section IV states the main result of the paper: it begins with a detailed stability analysis of the tracking error
(Sec. IV-A) and ends up with the main theorem which relies on singular perturbation analysis and Kelemen's stability result (Sec. IV-B).

- A short summary of main notations is available at the end of the paper in Table I.

\section{Summary OF Model-Free Control}

Model-free control is a very recent approach to nonlinear control that has been introduced in [2], (Refer to [3] for a thorough presentation). A first industrial and convincing application is reported in [13].

\section{A. Brief Introduction to Mikusiński's Operational Calculus}

A reader not familiar with advanced commutative algebra [14] can skip the reading of this section and go through the end of the communication just with usual knowledge of Laplace transformation [15]. This Section is devoted to a brief introduction to the operational calculus that has been introduced by Jan Mikusiński ${ }^{1}$.

a) Class $\mathcal{K}:$ A function ${ }^{2}\{f(t)\}:[0,+\infty[\longrightarrow \mathbb{C}$ is said to belong to class $\mathcal{K}$ if:

1) it has at most a finite number of points of discontinuity in every finite interval;

2) the integral
every $t>0$. $\int_{0}^{t}|f(\tau)| d \tau$ has a finite value for

b) Convolution: Given $f, g$ two elements of $\mathcal{K}$, their convolution is defined as

$$
\begin{aligned}
(f \star g)(t) & =\int_{0}^{t} f(t-\tau) g(\tau) d \tau \\
& =\int_{0}^{t} f(\tau) g(t-\tau) d \tau
\end{aligned}
$$

In the sequel, the " $\star$ " symbol for convolution is omitted.

c) Integral operator: According to the definition of convolution we have:

$$
\{1\}\{f(t)\}=\left\{\int_{0}^{t} f(\tau) d \tau\right\}
$$

And the function $\{1\}$ plays the role of integral operator and can also be denoted as $\mathfrak{l}$.

\footnotetext{
${ }^{1}$ Jan Mikusiski (April 3, 1913 Stanisaww July 27, 1987 Katowice) was a Polish mathematician.

${ }^{2}$ We use here the notation introduced by Mikusiński himself to denote functions.
} 
d) Cauchy formula: With the notations of operational calculus it is easy to establish the Cauchy formula:

$$
\begin{gathered}
\int_{0}^{t} \int_{0}^{t_{\kappa}} \ldots \int_{0}^{t_{3}} \int_{0}^{t_{2}} f\left(t_{1}\right) d t_{1} d t_{2} \ldots \ldots d t_{\kappa-1} d t_{\kappa} \\
=\int_{0}^{t} \frac{(t-\tau)^{\kappa-1}}{(\kappa-1) !} f(\tau) d \tau
\end{gathered}
$$

e) Construction of the operators: It is possible to show that $(\mathcal{K},+, \star)$ is a commutative ring, moreover, by Titchmarsh Theorem [16], this ring is integral domain and it is possible to define "an operation inverse to convolution" and construct the field of fraction of this ring.

Namely, denote as $a / b$ the solution of the convolution equation $a=b c, a, b \in \mathcal{K}$. The solution $c$ is not always a function of $\mathcal{K}$ and will be named an operator.

f) Differential operator: Denote as $\mathfrak{s}$ the operator defined as the inverse (w.r.t. to convolution) to $\mathfrak{l}$, i.e.

$$
\mathfrak{s}=\frac{1}{\mathfrak{l}}
$$

This operator has the following property: If a function $\{f\} \in \mathcal{K}$ has a continuous derivative $\{\dot{f}\}$, then

$$
\mathfrak{s}\{f\}=\{\dot{f}\}+f(0)
$$

g) Operational form of a function: One calls operational form of a function its expression in terms of the differential operator $\mathfrak{s}$. Example:

$$
\begin{aligned}
\{t\} & =\frac{1}{\mathfrak{s}^{2}} \\
\left\{\frac{t^{k}}{k !}\right\} & =\frac{1}{\mathfrak{s}^{k+1}} \\
\{\exp (-a t)\} & =\frac{1}{\mathfrak{s}+a}
\end{aligned}
$$

which mimics usual Laplace transforms.

Calculations of estimators rely on the operational calculus of Mikusiński (see [16]).

\section{B. A Short Recall on Algebraic Estimators}

Firstly, we recall basics of derivative estimation. Interested reader might refer to [17] for a complete presentation. We consider a time signal $v$ that is available through a measurement $v_{m}$ corrupted by some additive noise $\varpi$, i.e. $v_{m}=v+\varpi$. The objective is to estimate some time derivatives of signal $v$, up to a finite order of derivation, from its measurement $v_{m}$ observed on a given time interval.

The Taylor expansion of $v$ around 0 reads:

$$
v(\tau)=\sum_{n=0}^{\infty} v^{(n)}(0) \frac{\tau^{n}}{n !}
$$

Approximate $v(t)$ in the interval $[0, T], T>0$, by the polynomial

$$
v_{N}(\tau)=\sum_{n=0}^{N} v^{(n)}(0) \frac{\tau^{n}}{n !}
$$

of degree $N$. The operational ${ }^{3}$ analogue (see [16]) $\Upsilon_{N}$ of $v_{N}$ is given by:

$$
\Upsilon_{N}=\frac{v(0)}{\mathfrak{s}}+\frac{\dot{v}(0)}{\mathfrak{s}^{2}}+\cdots+\frac{v^{(N)}(0)}{\mathfrak{s}^{N+1}}
$$

It is possible to isolate each coefficient $v^{(i)}(0)$ appearing in the previous expression by applying a convenient operator to $\Upsilon_{N}(\mathfrak{s})$ (See [17] for details ${ }^{4}$. Indeed:

$$
\begin{aligned}
& \forall i=0, \ldots, N \text {, } \\
& \frac{v^{(i)}(0)}{\mathfrak{s}^{2 N+1}}=\frac{(-1)^{i}}{N !(N-i) !} \cdot \frac{1}{\mathfrak{s}^{N+1}} \cdot \frac{d^{i}}{d \mathfrak{s}^{i}} \cdot \frac{1}{\mathfrak{s}} \\
& \cdot \frac{d^{N-i}}{d \mathfrak{s}^{N-i}}\left(\mathfrak{s}^{N+1} \Upsilon_{N}(\mathfrak{s})\right)
\end{aligned}
$$

One obtain in the time domain the expression of $v^{(i)}(0)$ as:

$$
v^{(i)}(0)=\int_{0}^{T} P(\mu ; T) v_{N}(\mu) \mathrm{d} \mu
$$

where $P(\mu ; T)$ is a polynomial in $\mu$ and $T$. Notice that (3) gives the calculation of $y^{(i)}(0)$ from an integral on the time interval $[0, T]$ for a given small $T>0$. As $\left.\frac{d^{i} v(t-\mu)}{d \mu^{i}}\right|_{\mu=0}=$ $(-1)^{i} v^{(i)}(t)$ it is possible to express $v^{(i)}(t)$ as an integral which involves values of $v_{N}$ on the time interval $[t-T, t]$ :

$$
v^{(i)}(t)=(-1)^{i} \int_{0}^{T} P(\mu ; T) v_{N}(t-\mu) d \mu
$$

A simple estimator of the derivative $v^{(i)}(t)$ is then obtained from the noisy signal $v_{m}$ by:

$$
\left\langle\left\langle v^{(i)}\right\rangle\right\rangle_{T}^{\mathbf{c}}(t)=(-1)^{i} \int_{0}^{T} P(\mu ; T) v_{m}(t-\mu) d \mu
$$

which is deduced from (3) by replacing $v_{N}$ by $v_{m}$ in (4). Note that the integral operation plays the role of low-pass filter and reduced the noise that corrupts $v_{m}$. The choice of $T$ results in a trade-off: the larger is $T$, the smaller is the effect of the noise (the larger is $T$ the better is integrals low pass filtering) and the larger is the error due to truncation.

In practice, the integral appearing in (5) is calculated with a numerical integration method (Simpson,...) and the estimator $\left\langle\left\langle v^{(i)}\right\rangle\right\rangle_{T}^{\mathbf{c}}(t)$ is thus evaluated at each sample time $t_{k}=k \cdot T_{s}$, $k=0,1, \ldots$ Let denotes $T_{s}$ the sampling period and for any time function $f$ denotes as $f[k]$ its time-samples at period $T_{s}$, i.e.:

$$
f[k]=f\left(k . T_{s}\right), \quad k \in \mathbb{Z}
$$

\footnotetext{
${ }^{3}$ Reader not familiar with operational calculus can just think in terms of Laplace transform to understand the development of the derivatives estimators.

${ }^{4}$ Note that those operators are not unique, we have chosen here to use the ones with the least order of integration for the sake of simplicity of the presentation.
} 
With these notations, the discrete-time approximation of the estimator (5) is simply a discrete sum that can be rewritten as:

$$
\left\langle\left\langle v^{(i)}\right\rangle\right\rangle_{T_{s}, n_{s}}^{\mathbf{d}}[k]=\sum_{j=0}^{n_{s}} w(j) P\left(j T_{s} ; n_{s} T_{s}\right) v_{m}[k-j]
$$

with $n_{s}$ the number of samples used in the time window and $n_{s} T_{s}=T$ and the $w(j)$ 's are the weight associated with the used numerical integration method.

\section{Model-Free Control}

1) Problem statement: Assume we have a plant (see Fig. 1) and that one wants to control the output by mean of the control input, possibly in the presence of a disturbance $d$ acting on the system (effect of a load, noise...). The control input is denoted by $u$, the output is denoted as $y$ and the disturbance is denoted by $d$. The control objective can, in many situation be expressed in terms of a reference trajectory $t \mapsto y^{\star}$, that represents a desired behavior of the output of the system

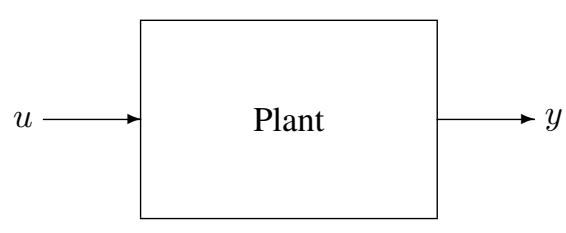

Fig. 1. To-Be-Controlled System

Assume that we do not know any control model of the system of Fig. 1. This is a case in many situations such as:

- the model is not well developed or not writable in a usual manner to be tractable by well-established control framework (for example models described by partial differential equation are not tractable by most of control methodologies);

- the known model is not easily identifiable or the identification of coefficient gives results with low confidence;

- the system is affected with strong disturbance that are difficult to track in any model.

The tracking problem can be also expressed as the asymptotic convergence to 0 of the tracking error $\epsilon$ defined by:

$$
\epsilon=y-y^{\star}
$$

for some non-zero initial condition $\epsilon(0) \neq 0$ and under the action of various disturbance.

2) Original Presentation : the Discrete-Time Version of Model-Free Control:

Remark 1: For the sake of simplicity, the presentation of this work is led with a single-input single-output system, i.e., $u(t) \in \mathbb{R}$ and $y(t) \in \mathbb{R}$. The general multi-variable case will be developed in a future publication.
A quite recent work has proposed a completely new solution to the problem presented in Sec. II-C1 and have named it precisely model-free control as it does not relies on any modeling of the to-be-controlled plant. This new control strategy has been introduced for the first time in [2] and further developed, with many application examples, in [3].

The control law that has been proposed by [2] can be summarized as follows: Introduce an auxiliary numerical model

$$
y^{(\nu)}=F+\alpha u
$$

where:

- $\quad \alpha \in \mathbb{R}$ is an non-physical constant design parameter;

- $F$ represents all that is unknown in the input-output behavior of the system;

- the order $\nu$ is also a design parameter of the numerical model (8) that can be arbitrarily chosen by the designer.

Although this can be confusing at first sight, Eqn. (8) should not be mixed up with a "black-box" identified model of the plant of Fig. 1. In model-free control, Eqn. (8) is updated at each sampling time from the knowledge of the input-output behavior of the unmodeled plant in order to estimate the unknown quantity $F$ and use it in the proposed control law (see below). With the notations introduced in Sec. II-B the estimation of $F$ at sample $k$ (i.e. at time $t_{k}=k . T_{s}$ ) reads:

$$
\widetilde{F}[k]=\left\langle\left\langle y^{(\nu)}\right\rangle\right\rangle_{T_{s}, n_{s}}^{\mathbf{d}}[k]-\alpha u[k]
$$

where $\left\langle y^{(\nu)}\right\rangle_{T_{s}, n_{s}}^{\mathbf{d}}[k]$ is the estimation of the derivative of order $\nu$ of the measured output of the plant and $u[k]$ the control input applied to the system at sample time $k . T_{s}, k=0,1, \ldots$

Based on the numerical knowledge $\tilde{F}$ at each sample $k$ of the quantity $F$, the control for sampling period $k$ is calculated on (8) as a simple cancellation of the nonlinear terms $F$ plus a closed loop tracking of a reference trajectory $t \mapsto y^{\star}(t)$ :

$$
u[k+1]=\underbrace{-\frac{\tilde{F}[k]}{\alpha}}_{\text {NL Cancellation }}+\underbrace{\frac{y^{\star(\nu)}[k]-\Delta_{\nu-1}^{\iota}(\epsilon)[k]}{\alpha}}_{\text {Closed loop tracking }}
$$

where:

- $\quad \epsilon=y-y^{\star}$ (and $\epsilon[k]=y[k]-y^{\star}[k]$ for all $k \in \mathbb{Z}$ ) is the tracking error;

- $\Delta_{\nu-1}^{\iota}$ is a closed-loop feedback controller based on the tracking error and that depends on the derivatives of $\epsilon$ up to the order $\nu-1$ and on the integrals of $\epsilon$ up to the order $\iota \in \mathbb{N}$.

The shifting of one sample between the right- and left-hand side (this is indeed $u[k+1]$ and not $u[k]$ in the left hand side of (10)) is necessary in order to avoid any algebraic loop.

Remark 2: The controller is expressed by a functional in $\epsilon$ as it may involve integral terms. Consequently we write $\Delta_{\nu-1}^{\iota}(\epsilon)(t)$ to express its value at time $t \in \mathbb{R}$ and $\Delta_{\nu-1}^{\iota}(\epsilon)[k]$ for its value at sample time $k T_{s}, k \in \mathbb{Z}$. 
Remark 3: The controller $\Delta_{\nu-1}^{\iota}$ can be of various forms, not necessarily linear. It is not useful to precise a detailed form at this stage of the presentation. See below for more details.

Remark 4: Note that the term $-\frac{\widetilde{F}[k]}{\alpha}+\frac{y^{\star[\nu]}[k]}{\alpha}$ is also the "nominal control" in the "flatness-based" control of (8) (See also [18]-[20]). When the closed loop controller is of "PID" type, model-free control can be named as "intelligent PID" ( $i$ PID) (See [3]). This control scheme is summarized in Fig. 2.

In order to be completely clear on the fact that modelfree control does not rely on any model, one can rephrase the presentation of model-free control as follows: One can think to solve the control problem exposed in Sec. II-C1 as finding a control law to the plant of Fig. 1 in order that its controlled behavior mimics the one of the following linear input-output system:

$$
y^{(\nu)}=\alpha u
$$

Keep in mind that (11) is not a model nor any try of modeling of the to-be-controlled plant, but a numerical intermediary in the development of the control law.

One can legitimately ask why not to choose simply $\alpha=1$ in order to obtain a simple Brunovský canonical form [21] (a simple chain of $\nu$ integrators in series) instead of (11). Note that this can also be achieved by a simple rescaling of the control variable $u$. However, as it will appear later, $\alpha$ plays an important role in the the tuning of the model-free control law and it will be kept as a design parameter.

Recall that robust asymptotic tracking of the trajectory $t \mapsto$ $y^{\star}$ to system (11) can easily be achieved with the closed-loop control law of the form

$$
u(t)=\frac{y^{\star(\nu)}-\Delta_{\nu-1}^{\iota}(\epsilon)(t)}{\alpha}
$$

where $\Delta_{\nu-1}^{\iota}$ is an appropriate controller, i.e. the closed-loop error equation:

$$
\epsilon^{(\nu)}+\Delta_{\nu-1}^{\iota}(\epsilon)=0
$$

impress $\lim _{t \rightarrow+\infty} \epsilon(t)=0, \forall \epsilon(0)$ (see below for a choice that ensures this convergence). Recall that $\iota \in \mathbb{N}$ stands for the number of error integrals and $\nu \in \mathbb{N}$ the number of derivatives of $\epsilon$ involved in the control law (12).

Remark 5: In the linear case, it is always possible to find a linear controller $\Delta_{\nu-1}^{\iota}$ that ensures global asymptotic stability, i.e. convergence to 0 of the tracking error from any initial condition

As the real plant of Fig. 1 is far from being so trivial as the simple mono-variable linear system (11), however, one can easily understand the model-free control as a generalization of (12) by adding an adaptive compensation term. To this end, consider the time-varying quantity $\Omega$ defined as:

$$
\Omega(t)=y^{(\nu)}(t)-\alpha u(t)
$$

This quantity can be though as a "measure of difference" between the input-output behavior of the plant of Fig. 1 and the one of the dynamic system of model (11). Notice that moreover, as it is written in terms of $u$ and $y$ it can easily be estimated with the techniques recalled in Sec. II-B. Its estimation $\widetilde{\Omega}$, at sample time $k \cdot T_{s}$, reads:

$$
\widetilde{\Omega}[k]=\left\langle\left\langle y^{(\nu)}\right\rangle\right\rangle_{T_{s}, n_{s}}^{\mathbf{d}}[k]-\alpha u[k]
$$

Note that $\widetilde{\Omega}$ is piecewise constant as it is estimated only once per sample period $\left[k \cdot T_{s},(k+1) \cdot T_{s}[\right.$. Having this estimation on can add a compensation term in the control law (12) in order to help the to-be-controlled systems mimics the tracking of $y^{\star}$ by (11).

The model-free control is thus

$$
u[k+1]=\frac{y^{\star(\nu)}[k]-\Delta_{\nu-1}^{\iota}(\epsilon)[k]-\widetilde{\Omega}[k]}{\alpha}
$$

In order to well understand the sequel of the development consider:

- The constant coefficient $\alpha \in \mathbb{R}$ is a non-physical design parameter of the control law (see below);

- $\quad$ the time-varying quantity $\Omega$ represents all that must be compensated from input-output knowledge $u \mapsto y$ in order to mimic (11) after feedback. The compensation concerns the effect of perturbations, the ignorance of the model or the value of model parameters, the coupling with various other input or output of the system...

- the order $\nu \in \mathbb{N}$ in (16) is also a design parameter that can be arbitrarily chosen.

The complete control scheme is depicted in Fig. 2. Notice that although the plant is a continuous-time one, the control law is a sampled-control or discrete-time control: the estimators of $\Omega$ and the control $u$ are calculated once for each sample period.

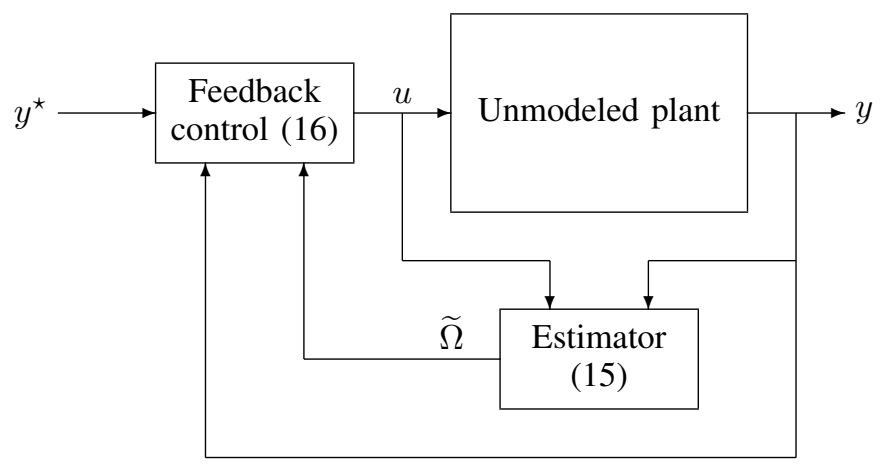

Fig. 2. Model-Free Control

Remark 6: Equation (16) is quite similar to (10), however the compensation term $\widetilde{\Omega}$ of (16) has a clear signification in terms of what can be compensated from the input-output view of any measurements of control systems. 


\section{CONTINUOUS-TIME REFORMULATION OF MODEL-FREE CONTROL}

\section{A. Euler Method Recall}

Consider the ordinary differential equation:

$$
\dot{x}(t)=f(x(t), t)
$$

where $x: I \longrightarrow \mathcal{X}, f: I \times \mathcal{X} \rightarrow \mathbb{R}^{n}, n \in \mathbb{N}, I=\left[t_{o}, t_{f}\right]$ is an interval of $\mathbb{R}$ and $\mathcal{X}$ an open subset of $\mathbb{R}^{n}$.

The simplest algorithm to approximate the solution to the Cauchy problem with initial condition $\left(t_{o}, x_{o}\right)$ associated with (17) is the Euler method that can be summarized as follows: Denote as $\left\{t_{0}, t_{1}, \ldots, t_{N}\right\}$ a subdivision of the time interval $I=\left[t_{o}, t_{f}\right]$, i.e. a strictly increasing sequence

$$
t_{o}=t_{0}<t_{1}<\cdots<t_{i}<\cdots<t_{N}=t_{f}
$$

and set

$$
h_{k}=t_{k+1}-t_{k}, \quad k=0,1, \ldots, N-1
$$

the successive steps of the algorithm. The approximated solution of (17) is denoted as $\tilde{x}$ and is iteratively calculated at $t=t_{k}$ according to:

$$
\begin{gathered}
\tilde{x}\left(t_{k+1}\right)=\tilde{x}\left(t_{k}\right)+h_{k} \cdot f\left(\tilde{x}\left(t_{k}\right), t_{k}\right) \\
k=0, \ldots, N-1
\end{gathered}
$$

This algorithm is initialized with:

$$
\begin{aligned}
t_{0} & =t_{o} \\
\tilde{x}\left(t_{0}\right) & =x_{o}
\end{aligned}
$$

Results of convergence and stability of this algorithm are well-established in numerical analysis and we will not repeat them here. Roughly speaking, the properties of the algorithm mainly relies on the maximum step $h=\max _{k}\left(h_{k}\right)$ (see [22] for details).

\section{B. Continuous Time Counterpart of the Control Law (16)}

The model-free control law (16) can be easily re-written in a different manner. To this end, recall that $y^{\star}=y-\epsilon$ so $y^{\star(\nu)}=$ $y^{(\nu)}-\epsilon^{(\nu)}$. Moreover from (14), $y^{(\nu)}(t)=\Omega(t)+\alpha u(t)$. Consequently:

$$
\begin{array}{r}
y^{\star(\nu)}[k]-\Delta_{\nu-1}^{\iota}(\epsilon)[k]-\widetilde{\Omega}[k] \\
=\alpha u[k]+\Omega[k]-\tilde{\Omega}[k]-\epsilon^{(\nu)}[k]-\Delta_{\nu-1}^{\iota}(\epsilon)[k]
\end{array}
$$

Moreover

$$
\Omega[k]-\tilde{\Omega}[k]=y^{(\nu)}[k]-\left\langle\left\langle y^{(\nu)}\right\rangle\right\rangle_{T_{S}, n_{s}}^{\mathbf{d}}[k]
$$

And finally the control reads:

$$
\begin{aligned}
u[k+1] & =u[k] \\
& +\frac{1}{\alpha}\left(y^{(\nu)}[k]-\left\langle\left\langle y^{(\nu)}\right\rangle\right\rangle_{T_{s}, n_{s}}^{\mathbf{d}}[k]\right. \\
& \left.-\epsilon^{(\nu)}[k]-\Delta_{\nu-1}^{\iota}(\epsilon)[k]\right)
\end{aligned}
$$

and comparing with (18), one can interpret (19) as the Euler algorithm associated to:

$$
\begin{aligned}
\dot{u}(t)= & \frac{1}{T_{s} \alpha}\left[y^{(\nu)}(t)-\left\langle\left\langle y^{(\nu)}\right\rangle\right\rangle_{T}^{\mathbf{c}}(t)\right. \\
& \left.-\epsilon^{(\nu)}(t)-\Delta_{\nu-1}^{\iota}(\epsilon)(t)\right]
\end{aligned}
$$

with initial condition $u(0)=0$ and $T=n_{s} T_{s}$. One see the influence of $\alpha$ : it can always be chosen in order that the time $\alpha T_{s}$ plays the role of a "small parameter", ensuring fast convergence of the control to the accurate value to achieve the control task. This will become more clear in the proof below.

\section{Stability ANALysis}

To our best knowledge every results of stability of solution of differential system rely on a model given by equations (see e.g. [23], [24] for a thorough survey). In order to analyze the stability of the closed loop we need to introduce a model of the system. Remember that this model is not used in the development of the control law (10) or (20). For the sake of simplicity, we only consider a single input single-output system (SISO) and the general case will be developped in a future publication. form:

Moreover, we assume that the model of the plant is of the

$$
y^{(\nu)}=f\left(y, \dot{y}, \ldots, y^{(\nu-1)}\right)+b u
$$

where $b \in \mathbb{R}^{\star}$ is a constant nonzero parameter and $f$ a convenient smooth vector field. A more general model will be considered in a future publication. Note that this is not a limitation to consider the same order $\nu$ in (21) and in (13) as this order can be arbitrarily chosen during the control law design in (10) or (20). The structure of model (21) is a controller-like form and this facilitate the error equation analysis.

Remark 7: The structure of the model (21) evades some class of control systems, especially the difficult case of nonminimum phase ones which, as everyone knows are difficult to control, even with Model-Free control [25].

\section{A. Error Equation Analysis}

The closed-loop (21)-(20)-(7) is given by:

$$
\begin{aligned}
y^{(\nu)}= & f\left(y, \dot{y}, \ldots, y^{(\nu-1)}\right)+b u \\
\dot{u}= & \frac{1}{T_{s} \alpha}\left[y^{(\nu)}(t)-\left\langle\left\langle y^{(\nu)}\right\rangle\right\rangle_{T}^{\mathbf{c}}(t)\right. \\
& \left.-\epsilon^{(\nu)}(t)-\Delta_{\nu-1}^{\iota}(\epsilon)(t)\right] \\
\epsilon= & y-y^{\star}
\end{aligned}
$$

For the stability analysis it is necessary to enter in the details of the controller $\Delta_{\nu-1}^{\iota}$. For the sake of simplicity we exhibit one which is linear although there are many nonlinear choices that are quite convenient. It worth to give some precision on the choice of the closed-loop controller $\Delta_{\nu-1}^{\iota}$ that renders the closed-loop system asymptotically stable. 
The tracking error is simply $\epsilon=y-y^{\star}$ and denotes as $\chi_{i}$, $i=1,2, \ldots, \iota$, the $i$-st integral of $\epsilon$, namely:

$$
\chi_{i}(t)=\underbrace{\int_{0}^{t} \int_{0}^{t_{i}} \cdots \int_{0}^{t_{3}} \int_{0}^{t_{2}}}_{i \text { integrals }} \epsilon\left(t_{1}\right) d t_{1} \ldots d t_{i}
$$

Among the many possible choices of stabilizing controller, linear ones exist and $\Delta_{\nu-1}^{\iota}$ can be chosen as:

$$
\begin{aligned}
\Delta_{\nu-1}^{\iota}(\epsilon)= & a_{-\iota} \chi_{i}+\cdots+a_{-1} \chi_{1} \\
& +a_{0} \epsilon+a_{1} \dot{\epsilon}+\cdots+a_{\nu-1} \epsilon^{(\nu-1)}
\end{aligned}
$$

One can renders the equation (13) globally asymptotically stable if the characteristic polynomial associated with this equation, namely:

$$
\mathcal{P}_{\Delta_{\nu-1}^{\iota}}(\lambda)=\lambda^{\nu+\iota}+a_{\nu-1} \lambda^{\nu+\iota-1}+\cdots+a_{1-\iota} \lambda+a_{-\iota}
$$

is Hurwitz, i.e. has all roots with strictly negative real part.

Eliminating $y=\epsilon+y^{\star}$ and all its derivatives in (22) leads to a first-order differential system that couples the variables: $\chi_{\iota}, \chi_{\iota-1} \ldots, \chi_{1}, \epsilon, \dot{\epsilon}, \ldots \epsilon^{(\nu-1)}$ and is excited by the quantities $y^{\star}, \dot{y}^{\star}, \ldots, y^{\star(\nu)}$, and $\left\langle\left\langle y^{(\nu)}\right\rangle_{T}^{\mathbf{c}}\right.$. In full details, the equations reads:

$$
\begin{aligned}
\frac{d \chi_{\iota}}{d t} & =\chi_{\iota-1} \\
& \vdots \\
\frac{d \chi_{1}}{d t} & =\epsilon \\
\frac{d \epsilon}{d t} & =\dot{\epsilon} \\
& \vdots \\
\frac{d \epsilon^{(\nu-1)}}{d t} & =\epsilon^{(\nu)} \\
\frac{d \epsilon^{(\nu)}}{d t} & =f\left(\epsilon+y^{\star}, \ldots, \epsilon^{(\nu-1)}+y^{\star}(\nu-1)\right) \\
\dot{u} & =\frac{1}{T_{s} \alpha}\left[y^{\star(\nu)}-\left\langle\left\langle y^{(\nu)}\right\rangle\right\rangle_{T}^{\mathbf{c}}-\Delta_{\nu-1}^{\iota}(\epsilon)\right]
\end{aligned}
$$

Which is a differential system of equation,

The last equation (24i) exhibits a "small parameter", namely $T_{s} \alpha$, that ensures a fast convergence of $u$ to the desired control. The complete stability analysis relies on the arguments of Kelemen's theorem [6].

\section{B. Proof of Stability}

Before to state the main result of this paper, we need to introduce some more vocabulary.

We will say that the stabilizing controller $\Delta_{\nu-1}^{\iota}$ ensures the tracking of the ideal system (13) with a sufficient margin if there exits $a>0$ such that all the roots $\lambda_{l}, l=1, \ldots, \nu+\iota$ of the associated ${ }^{5}$ characteristic polynomial $\mathcal{P}_{\Delta_{\nu-1}^{\iota}}$ (see (23)) satisfies

$$
\operatorname{Re}\left(\lambda_{l}\right)<-a, \quad l=1, \ldots, \nu+\iota
$$

\footnotetext{
${ }^{5}$ In case of a linear controller, see (23), in nonlinear case just take the characteristic polynomial of the linearized controller around 0 .
}

We will say that the reference trajectory $t \mapsto y^{\star}$ is quiet enough it there exist $\delta>0, \tau>0$ such that:

$$
\forall t \geqslant 0, \forall i=0, \ldots, \nu, \quad \frac{1}{\tau} \int_{t}^{t+\tau}\left|y^{\star(i)}(\mu)\right| d \mu<\delta
$$

One says that the tracking of the trajectory $t \mapsto y^{\star}$ is asymptotically stable [26] if the tracking error is bounded, i.e. there exists $\epsilon_{o}>0$ such that

$$
\sup _{t \in[0,+\infty[}|\epsilon(t)|<\epsilon_{o}
$$

and moreover

$$
\lim _{t \rightarrow+\infty} \epsilon(t)=0
$$

The stability result can now be stated as follows:

Theorem 1: Provided that:

- the trajectory $t \mapsto y^{\star}$ is quiet enough;

- the stabilizing controller $\Delta_{\nu}^{\iota}$ ensures the robust tracking of (13) with sufficiently margin;

- the initial tracking error $\epsilon(0)$ is sufficiently small.

Then the model-free control law (16) that the to-be-controlled system ensures the asymptotically stable tracking of the trajectory $t \mapsto y^{\star}$.

The hypotheses of this theorem are quite standard compared with the ones in the huge literature on trajectory tracking.

Proof: The proof proceed with an application of Kelemen [6]-[8] with a conjunct application of the singular perturbation theory [5], [27], [28] on the differential system (24a)(24i): The small parameter $\alpha T_{s}$ appearing in the last equation (24i) ensures fast convergence of $u$ to its "good value" $u^{*}$. The latter, according to a steady state analysis, expresses as:

$$
u^{\star}=-\frac{f\left(y^{\star}, \ldots, y^{\star(\nu-1)}\right)}{b}
$$

Stability of the tracking is easily proven by a standard application of Kelemen's result. Notice that in practice $u^{*}$ cannot be calculated as the model of the system is not known; the estimator involved in (15) do the job in an indirect manner.

\section{Conclusion}

This paper establishes that model-free control produces an asymptotically stable trajectory tracking for nonlinear systems under mild hypothesis. This proof also gives insight to the tuning of model-free control laws.

This result is easily transportable to multi-input multioutput systems.

Model-free control have been successfully applied in many practical situation before this proof was developed. This results should give some confidence to control practitioners for the applicability of this framework. One also hope that this could facilitate the introduction of this new methodology in modern teaching of control. 


\section{ACKNOWLEDGMENTS}

The authors would like to thank A. S. Lucas for her helpful hints and benevolent encouragements during the preparation of this work.

The author humbly thanks the thousands of volunteers who give their time to develop free softwares or open hardwares; they actively contribute to protect the intellectual heritage of Humanity.

\section{NOTATIONS}

\begin{tabular}{|c|c|}
\hline$f[k]$ & Sample $k$ of time function $f$ \\
\hline$\left\langle\langle v\rangle_{T}^{\mathbf{c}}\right.$ & Continuous-time estimation of $v$ \\
\hline$\langle v\rangle_{T_{s}, n_{s}}^{\mathbf{d}}$ & discrete-time estimation of $v$ \\
\hline$T$ & $\begin{array}{l}\text { Time span used in } \\
\text { continuous-time estimators }\end{array}$ \\
\hline$T_{s}$ & Sample period \\
\hline$n_{s}$ & $\begin{array}{l}\text { Number of sample involved } \\
\text { in discrete-time estimators }\end{array}$ \\
\hline
\end{tabular}

TABLE I. MAIN NOTATIONS

\section{REFERENCES}

[1] N. Wiener, Cybernetics, or Control and Communication in the Animal and the Machine. New York, NJ: MIT Press/John Wiley and Sons, 1948, see [?] for a recent French translation.

[2] M. Fliess, C. Join, M. Mboup, and H. Sira-Ramìrez, "Vers une commande multivariable sans modèle," in Proc. Conférence internationale francophone d'automatique (CIFA'06), 2006. [Online]. Available: http://hal.inria.fr/inria-00001139/fr/

[3] M. Fliess and C. Join, "Model-free control and intelligent PID controllers: towards a possible trivialization of nonlinear control?' in 15th IFAC Symposium on System Identification (SYSID 2009). Saint-Malo France: IFAC, 2009. [Online]. Available: http://hal.inria.fr/ inria-00372325/en/

[4] M. Fliess, C. Join, and H. Sira-Ramírez, "Non-linear estimation is easy," International Journal of Modelling, Identification and Control (IJMIC), vol. 4, no. 1, pp. 12-27, 2008. [Online]. Available: http://hal.inria.fr/inria-00158855/fr/

[5] F. Hoppensteadt, "Singular perturbations on the infinite interval," Transactions of the American Mathematical Society, vol. 123, pp. 521-535, 1966.

[6] M. Kelemen, "A stability property," IEEE Trans. Autom. Control, vol. 31, pp. 766-768, 1986.

[7] A. L. Lawrence and W. J. Rugh, "On a stability theorem for nonlinear systems with slowly varying inputs," IEEE Trans. Autom. Control, vol. 35, pp. 860-864, 1990 .

[8] H. K. Khalil and P. V. Kokotović, "On stability properties of nonlinear systems with slowly varying inputs," IEEE Trans. Autom. Control, vol. 36, pp. 229-229, 1991.

[9] V. Hagenmeyer and E. Delaleau, "Exact feedforward linearization based on differential flatness," Internat. J. Control, vol. 76, no. 6, pp. 537-556, 2003.

[10] _ "Robustness analysis of exact feedforward linearization based on differential flatness," Automatica J. IFAC, vol. 39, pp. 1941-1946, 2003
[11] _ _ "Continuous-time non-linear flatness-based predictive control: an exact feedforward linearisation setting with an induction drive example," Internat. J. Control, vol. 81, no. 10, pp. 1645-1663, 2008.

[12] — "Robustness Analysis with Respect to Exogenous Perturbations for Flatness-Based Exact Feedforward Linearization," IEEE Trans. Automat. Control, 2010, (See [?] for an extended version).

[13] C. Join, J. Masse, and M. Fliess, "Étude préliminaire d'une commande sans modèle pour papillon de moteur," Journal européen des systèmes automatisés (JESA), vol. 42, pp. 337-354, 2008. [Online]. Available: http://hal.inria.fr/inria-00187327/fr/

[14] B. L. van der Waerden, Modern Algebra. Springer, 1950, vol. I \&ĨI.

[15] G. Doetsch and W. Nader, Introduction to the Theory and Application of the Laplace Transformation. Springer, 1974.

[16] J. Mikusiński, Operational Calculus. Oxford: Pergamon Press, 1959.

[17] M. Mboup, C. Join, and M. Fliess, "Numerical differentiation with annihilators in noisy environment," Numerical Algorithms, vol. 50, no. 4, pp. 439-467, 2008. [Online]. Available: http: //hal.inria.fr/inria-00319240/fr/

[18] M. Fliess, J. Lévine, P. Martin, and P. Rouchon, "Flatness and defect of nonlinear systems: Introductory theory and examples," International Journal of Control, vol. 61, pp. 1327-1361, 1995.

[19] V. Hagenmeyer and E. Delaleau, "Exact feedforward linearization based on differential flatness," Internat. J. Control, vol. 76, pp. 537-556, 2003.

[20] _ _ "Robustness analysis of exact feedforward linearization based on differential flatness," Automatica J. IFAC, vol. 39, pp. 1941-1946, 2003.

[21] P. Brunovský, "A classification of linear controllable systems," Kybernetika, vol. 3, no. 6, pp. 173-188, 1970.

[22] W. H. Press, S. Teukolsky, W. Vetterling, and B. Flannery, Numerical Recipes. The Art of Scientific Computing. New York: Cambridge University Press, 2007.

[23] M. Vidyasagar, Nonlinear Systems Analysis. Prentice-Hall, 1978.

[24] H. H. Khalil, Nonlinear systems, 2nd ed. Upper Saddle River: Prentice hall, 2002.

[25] M. Fliess and C. Join, "Commande sans modèle et commande à modèle restreint," e-STA, vol. 5, no. 4, pp. 1-23, 2008. [Online]. Available: http://hal.inria.fr/inria-00288107

[26] J.-P. Demailly, Analyse numrique et quations diffrentielles, 3rd ed. Presses Universitaires de Grenoble, 2006.

[27] N. Fenichel, "Persistence and smoothness of invariant manifolds for flows," Indiana Univ. Math. J., vol. 21, pp. 193-226, 1971.

[28] E. N. Lorenz, "On the existence of a slow manifold," Journal of Atmospheric Science, vol. 43, pp. 1547-1557, 1986. 